\title{
An Effective Healthcare Management Frame work in Cloud Computing Environment
}

\author{
V. Gurunatha Prasad, R. Surya Prakash, H. Sathish Kumar, R. Raj Kumar, and N.Ch.S.N. Iyengar
}

\begin{abstract}
Cloud Computing the technology of today, made our life comfortable and easy by making most of the services which are on-demand, were brought down to our fingertip with Scalability, High performance, Low Cost. The most remarkable change made by cloud computing is that it made management and allocation of resources simple and federal. In the list of management services offered by the cloud, Health care services play a vital role. Though the connectivity issues in these kinds of services were solved by the cloud, data management issues like data storage, data retrieval, and data security still exists. Hence this current proposal speaks about establishing an enhanced cloud environment targeted for Healthcare Services.
\end{abstract}

Keywords---SaaS, PaaS, IaaS, EHR (Electronic Healthcare Records), EMR (Electronic Medical Record), MRU(Medical Records Unit)

\section{INTRODUCTION}

$\mathrm{C}$ LOUD computing an advanced model of networking with particular protocols that easy the way of utilizing and accessing the resources remotely, this model has warped the computing field technologies, enterprises has jumped into developing the technologies based on the cloud. Cloud computing has several types of services but the most basic services are Software as a service (SaaS), Platform as a service (PaaS), and Infrastructure as a service (IaaS), this provides the type of service to the consumers that let them to store and access data globally. Cloud has several features [1], [2] like adaptability, connectivity, reduction of cost, security, privacy and high performance which are essential to healthcare systems. In health care [3] more researches, practices and several tools needed to harvest knowledge from the patient's data, here hospitals [4] are the backbone of the country aims at curing disease and preventing them. Providing the health care [5] services as information through cloud, rebuilt the method of accessing the patient's records and that will based on patient-centric. Healthcare organization stores the patient's records locally, as images, scan, blood test reports, and so on, this increases the data size to more than petabytes [6] and makes them a complex situation to manage the data without the required resources.Handling their medical data for patient is complex and complex to the organization to, but organization stores patient records locally and never let the patient to view their own records from outside of the organization.

Gurunatha Prasad, R. Surya Prakash, and H. Sathish Kumar, are PG Students and with VIT University, Vellore -632014 Tamilnadu, India

R. Rajkumar is Asso. Professor at SCSE VIT University, Vellore -632014 Tamilnadu, India

Dr. N. Ch. S. N. Iyengar is Senior Professor, Professor at SCSE VIT University, Vellore -632014 Tamilnadu, India.
Hospitals spend lots of money to establish the Medical Records Unit (MRU) to maintain and store the patient's data. In case of emergency state patients medical records may be needed to the other hospitals for further treatment where EMR will not allow them to access and it is not sure patient may have his treatment records with him, this could lose many lives. Cloud provides the possibility to enhance [7] the way to integrate data available in several devices in healthcare management and reduces the expenses. Electronic healthcare record (EHR) has confidential healthcare data regarding the patient which is more important for [8] patient privacy has to be maintained, as the size of the records increase big data on biomedical data [9] and healthcare studies utilized for research. Providing methods and protocols to store the medical data in cloud and integrate them in to several hospitals to access the records when needed for the patient.

\section{LITERATURE REVIEW}

Cloud computing easy the way of accessing the data remotely and it reduces the complexity of handling the medical records. In [1] has focused on home healthcare system which is based on cloud computing, they have designed an architecture that is on patient-centric control, the system integrates security and privacy of the patients healthcare data provided a comprehensive methodology based on the cloud infrastructure.In [2] have presented an assisted I-living architecture for healthcare where independent persons work together in a dependable environment that ensure low-cost and secure, proposed an approach to collect and maintain encrypted records.In [3] have provided groundwork on personalized healthcare with big data driven approach to derive patient centric outcomes, this makes approaches to analyze the patients data records and form it in different centric approach such as disease-centric, the application reduces re-admission fees and provide meaning full use.In [4] has analyzed the crises and risk for healthcare providers with the cloud infrastructure impact, the analysis is taken in such a way of human aspects and IT-related that incorporates in overall view of risk management.In [5] have designed a framework to adopt electronic healthcare into cloud computing infrastructure with National cloud framework (NCF) that easy the accessing of patients healthcare data, and discussed the challenges on electronic healthcare methods to solve the issues with NCF.

\section{III.PROPOSED METHOD}

The Healthcare Cloud (HCC) completely comes under the control of the cloud manager who maintains over the cloud. The cloud manager is responsible for the communication, data 
sharing and security procedures in the cloud environment. The Cloud Manager controls the cloud by managing the components around it.

Fig. 1 The system architecture is clear and straightforward in describing the flow of the system. The patients or end users are connected to the cloud through the hospitals. The Cloud is controlled by the Trusted Cloud Manager. The core functional components of this specific system are the Key Store and the Data Store. The Key store is accessed through authentication services and Data store is accessed through applications and services which are data dependent. Structure and components of both key store and data store is discussed in detail in the below.

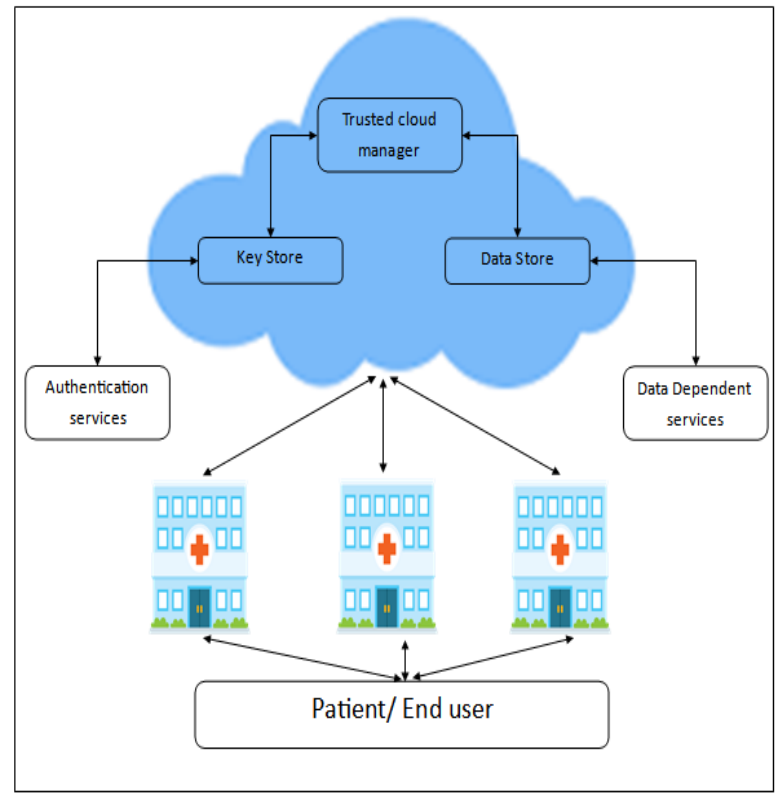

Fig. 1 System Architecture

\section{A. Data Store}

Data Store is a simple data storage system in the cloud which is divided and accessed in two different servicing methods, namely (i) Block Storage and (ii) Object Storage. The Block Storage Service provides privileges and access methods via tools and utilities written for it, through the block storage drivers, which allows accessing the complete memory in the unit of blocks of a specific size. Here in this proposed system block storage is used to store multimedia enabled medical or healthcare data.

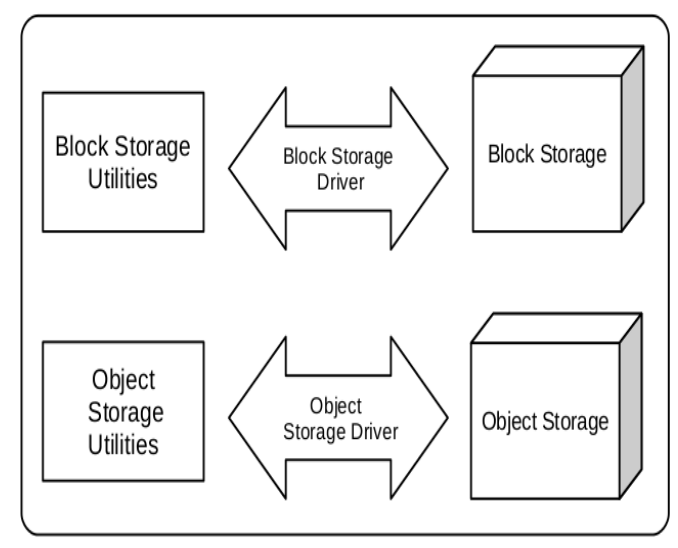

Fig. 2 Components of Data Store

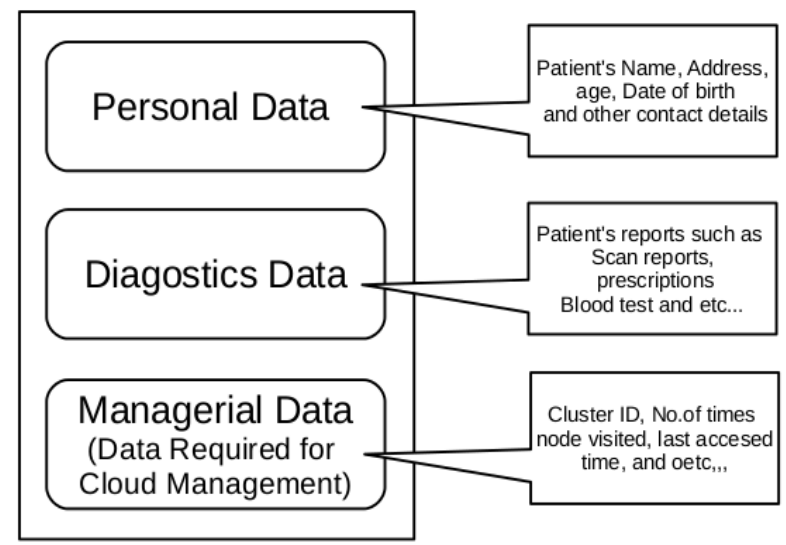

Fig. 3 Structure of the Basin

In the same way the Object Storage components of the data store allows us to access the data in terms of objects, which are of different sizes. These objects of the healthcare cloud are used to handle data that varies with from patient to patient which may more probably be of different sizes. We would like to call these data object in the cloud as Basin(s). There cloud is built with utilities that will build the basin in a specified format in order to make the implementation more sensible and objective in nature.

\section{A. Basin Generation}

When the initial authentication process gets completed, the Cloud manager now creates a basin for the patient. The Basin consists of three parts, that stores (1) Patient's Personal Data, (2) Patient's Diagnostic Data, (3) Managerial Data (HCC Purpose) as specified below. The Cloud Manager now recognizes the personal data, diagnostic data and stores them in the respective part of the basin. The Fig. 3 given below clearly describes the structure of the basin and its essential blocks with their objectives.

\section{B. Key Store}

Key Store is a solid component of this model that is not just responsible for key management but also for Identity generation, Identity Management, Data Indexing and Access Authorization. The Fig. 4 given below clearly describes the components and functional utilities of the key store.

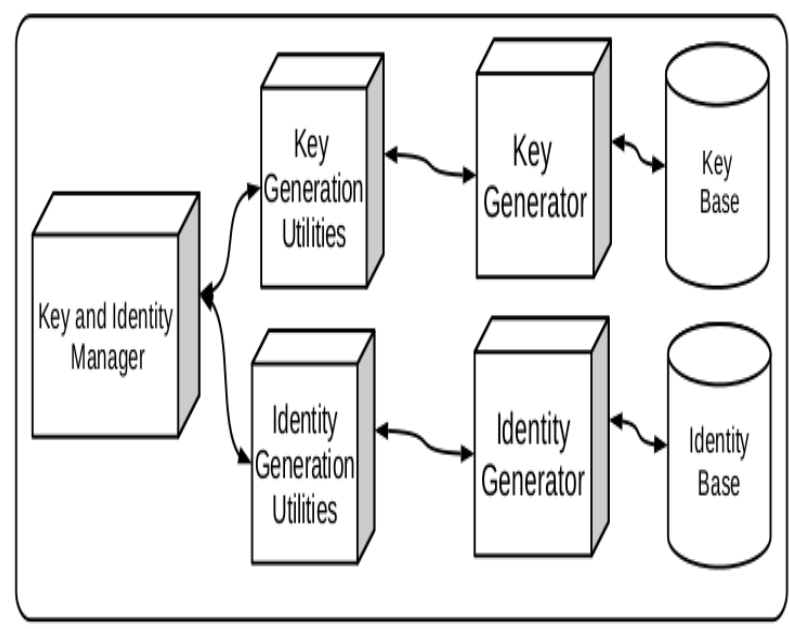

Fig. 4 Components of the key store 
The key and Identity Manager uses the key generation utilities to generate key sets in the programmed way and store them in the key base for future use. The process is eventually carried out with identity generation too. The role of Identity generator is not just generating IDs but also identify the data block for each and every specific ID allocated from the Identity Base. The Identity Manager also associates the keys generated with the ID and eventually to the data store, for the purpose of security through data encryption, where the data prevails.

\section{Protocol Flow}

This part of the proposed method is written to prove the coordination of components of the cloud with respect to the implementation. Here we describe some the phases involving the proposed components and their design to support the objective and enable security and elegance in the implementation.

\section{Protocol of Patient Registering in Healthcare Cloud:}

\author{
Hospital -> Cloud Manager: \\ Requesting for the session \\ Cloud Manager: \\ Hospital Authentication (Hospital ID) \\ IF (Hospital ID = Approved) \\ Start Session ; \\ ELSE \\ deny session;
}

\author{
Creating Patient's Basin in Cloud \\ Hospital -> Cloud Manager: \\ Patient Details \\ Cloud Manager: \\ Patient Detail Verification () \\ Patient ID Generation () \\ Create Basin () \{In specified Format\} \\ Hospital -> Cloud Manager: \\ Patient's Diagnosis Details \\ Cluster_Based_Indexing(Patient_ID, Diag_Data) \\ Cloud Manager -> Hospital: \\ Patient's ID \\ Hospital -> Cloud Manager: \\ Username and Password \{Received From Patient \} \\ Cloud Manager: \\ Generating Keys () \\ Cloud Manager -> Hospital: \\ Patient's Key Set \{Private Key and Public Key \} \\ Cloud Manager: \\ Update Identity Base (Patient_ID, Time Stamp)
}

The above protocol describes the patient registration process. The flow begins with session request for the hospital, to the Cloud Manager. After the hospital is authenticated the session is successfully established. With respect to the session the hospital sends the patient's data to the cloud manager. The details received is verified and an unique ID, private key and public key are generated by the key store and it communicates with the data store and a data basin is created,encrypted with the public key and indexed. The generated keysets are reported back to the hospital.

\section{Data Retrieval Process:}

Client -> Cloud Manager:

Patient ID, Username, Password

Cloud Manager:Patient Authentication (User Name,

Patient_ID, Password)

IF (Authentication Status $=$ SUCCESS)

Establish Session \{PID + Patient's PuK\}

ELSE

deny session

Client -> Cloud Manager:

Request For Data + Last Access Time

Cloud Manager:

Search Engine (Patient_ID, Last Access Time)

Identity Base(Patient_ID, Last Access Time)

WHILE(Patient_ID Index <>Patient_ID @ Last Access Time) Move Forth

RETURN Cluster ID, Basin Co-ordinates

\section{Cloud Manager:}

Retrieve Basin (Patient_ID)

Cloud Manager -> Client:

Encrypted Basin containing User Data

Client:

Decrypt Basin(Public Key, Private Key)

This specific search engine that retrieves the patient's data uses the last access time of the patient. The complete identity base has the LAT (last access time) entry for all the records. Based on the LAT the complete identity base is grouped. With selective group, the ID is cornpared and respective basin location for the patient is fetched. The location is sent to the data store and the encrypted basin is loaded at the client's end for decryption through patient's private key.

\section{CONCLUSION AND FUTURE WORK}

In this paper, we propose a framework to manage patient health records in secure way and protecting privacy of the patient. Here we discussed the proposed architecture briefly, we are carrying out the implementation process using the OpenStack Liberty which is elegant, powerful by build and supports the implementation with respect to visibility to internal components, source code as well as licensing. The modules and utilities described above are under development using python, in order to make the system build tightly integrated with the OpenStack environment. Research and development of the project is being carried out by us in VIT UNIVERSITY.

\section{REFERENCES}

[1]. M. Deng, M. Petković, M. Nalin, and I. Baroni, "A Home Healthcare System in the Cloud--Addressing Security and Privacy Challenges," IEEE International Conference onCloud Computing, pp. 549-556, July 2011.

http://dx.doi.org/10.1109/cloud.2011.108

[2]. Q. Wang, W. Shin, X. Liu, Z. Zeng, C. Oh, B. K. AlShebli, and L. Sha, "ILiving: An open system architecture for assisted living," IEEE International Conference onSystems, Man and Cybernetics, Vol. 5, pp. 4268-4275, October 2006.

http://dx.doi.org/10.1109/icsmc.2006.384805 
[3]. N. V.Chawla, and D. A. Davis, "Bringing big data to personalized healthcare: a patient-centered framework," Journal of general internal medicine, Vol. 28, No. 3, pp. 660-665, 2013. http://dx.doi.org/10.1007/s11606-013-2455-8

[4]. R. Glasberg, M. Hartmann, M. Draheim, G. Tamm, and F. Hessel, "Risks and crises for healthcare providers: the impact of cloud computing," The Scientific World Journal, 2014. http://dx.doi.org/10.1155/2014/524659

[5]. ChineduUkeje and O.A Oni, "The Adoption of a National Cloud Framework for Healthcare Delivery in Nigeria," Int. Journal of Engineering Research and Applications, Vol. 5, No. 3, pp. 61-68, 2015.

[6]. A. Umamakeswari, N. Vijayalakshmi, andT. Renugadevi, "Storage and Retrieval of Medical Images using Cloud Computing," Journal of Artificial Intelligence, Vol. 5, No. 4, pp. 207, 2012. http://dx.doi.org/10.3923/jai.2012.207.213

[7]. L. Bănică, and L. C. ŞTEFAN, "Cloud-powered e-Health,” Scientific Bulletin-Economic Sciences, Vol. 12, No. 1, pp. 26-38, 2013.

[8]. Y. A. A. S. Aldeen, M. Salleh, and M. A. Razzaque, "A comprehensive review on privacy preserving data mining," SpringerPlus, Vol. 4, No. 1, pp. 1-36, 2015. http://dx.doi.org/10.1186/s40064-015-1481-x

[9]. A. W. Toga, and I. D. Dinov, "Sharing big biomedical data," Journal of Big Data, Vol. 2, No. 1, pp. 1-12, 2015.

http://dx.doi.org/10.1186/s40537-015-0016-1

V. Gurunatha Prasad, R. Surya Prakash, and H. Sathish Kumar are students of M.C.A final year semester at school of information technology and engineering, VIT UNIVERSITY, Vellore, Tamil Nadu, India. Our area of interest is Cloud Computing, Internet of things, and Big Data.

R. Rajkumar is currently Associate Professor at School of Computing Science and Engineering at VIT University, Vellore-632014, Tamil Nadu, India. His research interests include mobile healthcare, and cloud computing. $\mathrm{He}$ has authored several research publications in reputed peer reviewed international journals.

N. Ch. S. N. Iyengar ( b.1961) is a senior Professor at School of Computer Science and Engineering at VIT University, Vellore, TN, India. His research interests include Distributed Computing, Information Security, Intelligent Computing, and Fluid Dynamics (Porous Media). He had much teaching and research experience with a good number of publications in reputed International Journals \& Conferences. He chaired many Intl. Conf. delivered Key note lectures, served as PC Member/Reviewer. He is Editorial Board member for many Int'l Journals like Int. J. of Advances in Science and Technology, of SERSC, Cybernetics and Information Technologies (CIT) -Bulgaria, Egyptian Computer Science Journal -Egypt, IJConvC of Inderscience -China, IJCA (USA) etc. ,Also Editor in Chief for International Journal of Software Engineering and Applications( IJSEA) of AIRCC, Advances in Computer Science (ASC) of PPH, Guest editor for "Cloud Computing and Services" IJCNS. 